\title{
Incidencia y características clínicas de pacientes trasplantados renales con infección y enfermedad por citomegalovirus en un centro de trasplante
}

\author{
Incidence and clinical characteristics of renal \\ transplanted patients with infection and disease by \\ cytomegalovirus in a transplant center
}

\author{
Kateir Contreras, María José Vargas, Julián Manrique, \\ Paola K. García, Martha Patricia Rodríguez, Camilo Alberto González \\ - Bogotá, D.C. (Colombia)
}

\section{Resumen}

Introducción: la infección viral más importante postrasplante renal es la infección por citomegalovirus (CMV), hay discrepancia entre centros y países en datos de incidencia de infecciónenfermedad en esta población de pacientes.

Diseño: se realiza un estudio observacional analítico, tomando una cohorte retrospectiva de pacientes mayores de 18 años, trasplantados renales de donante vivo o cadavérico entre el 2004 y 2015 con al menos seis meses de seguimiento.

Material y métodos: se realiza muestreo no probabilístico por conveniencia, se toman los datos de las historias clínicas de los pacientes trasplantados renales, calculando la densidad de incidencia de infección-enfermedad por CMV y se describen las características clínicas y demográficas de los pacientes que presentaron estas patologías.

Resultados: se analizaron 252 pacientes, encontrando $92.4 \%$ receptores con riesgo intermedio para CMV y 7.5\% con riesgo alto, ninguno fue de riesgo bajo. Se identificaron 19 casos, 13 con infección (5.1\%) y seis con enfermedad (2.3\%). El compromiso gastrointestinal fue el más frecuente. El tiempo promedio desde el momento del trasplante hasta la aparición de la infección-enfermedad fue de $417( \pm 479)$ y 650 días $( \pm 481)$, respectivamente. La tasa de infección fue de 10.08 casos por 1000 pacientes/año y la tasa de enfermedad de 5.88 por 1000 pacientes/año.

Conclusiones: la densidad de incidencia de infección-enfermedad por CMV en pacientes trasplantados renales fue de 10.08 casos y 5.88 casos por 1000 pacientes/año, respectivamente. Estas tasas son menores a las reportados en la literatura. Dada la baja frecuencia de eventos, no fue posible establecer factores de asociación. (Acta Med Colomb 2018; 43: 20-23)

Palabras clave: Citomegalovirus, leucopenia, trasplante renal, valganciclovir.

\footnotetext{
Abstract

Introduction: the most important viral infection after renal transplantation is cytomegalovirus (CMV) infection. There is a discrepancy between centers and countries in terms of incidence data of infection-disease in this population of patients.

Design: an analytical observational study was conducted, taking a retrospective cohort of patients older than 18 years old, kidney transplant recipients of living or cadaveric donors between 2004 and 2015, with at least 6 months of follow-up.

Material and methods: non-probability convenience sampling was done; data from the clinical records of the kidney transplant patients were taken, calculating the incidence density of CMV infection-disease and the clinical and demographic characteristics of the patients who presented these pathologies were described.
}

Dra. Kateir Contreras: Internista, Nefróloga, Unidad de Nefrología y Trasplante Renal, Hospital Universitario San Ignacio. Docente $A d$ Honorem Pontificia Universidad Javeriana; Dra. María José Vargas: Fellow Nefrología, Facultad de Medicina, Pontificia Universidad Javeriana; Julián Manrique: Estudiante de Medicina Pontificia Universidad Javeriana; Dra. Paola K. García: Jefe Unidad de Nefrología y Trasplante Renal, Hospital Universitario San Ignacio. Profesora Asistente, Pontificia Universidad Javeriana; Dra. Martha Patricia Rodríguez: Internista, Nefróloga, Unidad de Nefrología y Trasplante Renal, Hospital Universitario de San Ignacio. Profesora Asistente Pontificia Universidad Javeriana; Dr. Camilo Alberto González: Internista Nefrólogo, Unidad de Nefrología y Trasplante Renal Hospital Universitario San Ignacio. Docente Ad Honorem Pontificia Universidad Javeriana. Bogotá, D.C. (Colombia). Correspondencia: Dra. Kateir Mariel Contreras Villamizar, Bogotá, D.C. (Colombia)

E-mail:kamacovi@gmail.com Recibido: 12/XII/2016 Aceptado: 27/IX/2017 
Results: 252 patients were analyzed; $92.4 \%$ of recipients with intermediate risk for CMV and $7.5 \%$ with high risk were found. None of them had low risk. 19 cases were identified, 13 with infection $(5.1 \%)$ and 6 with disease $(2.3 \%)$. Gastrointestinal involvement was the most frequent. The average time from the time of transplant to the onset of the infection-disease was $417( \pm 479)$ and 650 days $( \pm 481)$, respectively. The infection rate was 10.08 cases per 1000 patients / year and the disease rate was 5.88 per 1000 patients/year.

Conclusions: the incidence density of CMV infection-disease in renal transplant patients was 10.08 cases and 5.88 cases per 1000 patients / year, respectively. These rates are lower than those reported in the literature. Given the low frequency of events, it was not possible to establish association factors. (Acta Med Colomb 2018; 43: 20-23).

Key words: Cytomegalovirus, leukopenia, kidney transplant, valganciclovir.

\section{Introducción}

El citomegalovirus (CMV) es la causa más frecuente de infecciones virales en el postrasplante renal, el CDC de Atlanta establece su prevalencia en $50-80 \%$ en adultos mayores de 40 años, con tendencia a aumentar con la edad (1). Su presentación en el postrasplante obedece en la mayoría de los casos, a la reactivación de la infección, favorecida por el estado de inmunosupresión del receptor. Se establece una condición de riesgo, dependiente del estado serológico (IgG para CMV), los receptores que tienen Ig $\mathrm{G}$ positiva $(\mathrm{R}+)$ son clasificados de riesgo intermedio para reactivación, los receptores Ig $\mathrm{G}$ negativa (R-) con donante Ig G negativa (D-) son de bajo riesgo y los R-con D+ son de alto riesgo. En Colombia un estudio realizado en Medellín documentó la presencia de Ig G positiva para CMV en $89 \%$ de los pacientes con trasplante renal y en $81 \%$ de los donantes (1). Un estudio recientemente publicado que evaluó donantes y receptores en el periodo 2010-2014, incluyó todo el territorio nacional y documentó seroprevalencia para CMV en donantes de $86.2 \%$ y en receptores de trasplantes renales de $91 \%$ (2), similar a otros países en vías de desarrollo. La distribución del riesgo en este estudio fue 7.3, 91.4 y $1.3 \%$ para alto, intermedio y bajo riesgo respectivamente (2).

Otros factores de riesgo identificados son: edad avanzada (>60 años), trasplante riñón-páncreas, antecedente de rechazo agudo y el uso de medicamentos como ciclosporina e inmunoglobulina antitimocito, la cual aumenta dos a cinco veces el riesgo (3).

La reactivación de infecciones ocurre frecuentemente entre el primero y cuarto mes, coincidiendo con las dosis más elevadas de terapia inmunosupresora; sin embargo, pasados los seis primeros meses puede ocurrir infección o enfermedad tardía (4). Los pacientes trasplantados desarrollan infección o enfermedad (5), el diagnóstico de infección es basado en la detección de CMV en sangre periférica (virus, proteínas virales o ácidos nucleicos) y el de enfermedad por la presencia de síntomas y signos clínicos.

En ausencia de medidas preventivas, la frecuencia de reactivación de infección es de 40-100\% y de enfermedad a $67 \%$ (6); por esto se diseñaron estrategias de prevención, siendo al momento las dos principales: profilaxis universal y terapia anticipada o preemptive (7). La profilaxis universal implica la administración de antivirales después de los 10 días del trasplante por un tiempo definido de tres a seis meses, ganciclovir y valganciclovir tienen similar efectividad (8) con la preferencia de este último, debido a su biodisponibilidad y vía de administración.

La literatura muestra diferentes porcentajes de infección y enfermedad, dependientes del medicamento que se haya usado para la inducción. Grecia reporta datos de infección de $12.8 \%$ y enfermedad $3.9 \%$ (6); modelos previos con pacientes de riesgo intermedio en Estados Unidos determinan infección en profilaxis universal $5.7 \%$ y en anticipada $55.5 \%$, con enfermedad en $6.3 \%$ de los casos en el primer año (9).

La importancia del estudio de esta entidad, no sólo radica en su alta prevalencia e incidencia, sino en la asociación que ha tenido con varios desenlaces adversos, derivados de la presentación de enfermedad o efectos indirectos asociados a la infección. El más frecuentemente descrito es el aumento en la incidencia de rechazo del injerto. También se asocia con fibrosis intersticial y atrofia tubular en los tres meses postrasplante; aumento del riesgo de infecciones asociadas como Candida, Pneumocystis jirovecci, Aspergillus y virus como Epstein Barr, herpes virus seis y ocho; mayor riesgo cardiovascular y estenosis de la arteria renal (5). Por último, puede producir un aumento de la mortalidad de 2.5-2.9 veces mayor en los pacientes trasplantados (3).

Es importante conocer estadísticas locales, por ello se realiza la descripción de las características clínicas de los pacientes trasplantados renales en nuestra institución que presentan infección- enfermedad por citomegalovirus y comparar nuestros resultados con los datos de otros grupos de trasplante tanto a nivel nacional como internacional dado que la incidencia y prevalencia tiene variación económica, geográfica y factores étnicos. Adicionalmente, no hay datos en nuestro país sobre la densidad de incidencia.

\section{Material y métodos}

Se realiza un estudio observacional analítico, tomando una cohorte retrospectiva de pacientes mayores de 18 años, trasplantados renales de donante vivo o cadavérico entre 2004 y 2015, con al menos seis meses de seguimiento. Los datos se obtuvieron de los registros de la historia clínica electrónica y de los resultados de los laboratorios que se 
les han practicado a los pacientes durante el seguimiento.

Se realizó el análisis descriptivo de las variables sociodemográficas y clínicas de la población del estudio. Para las variables categóricas se usaron medidas de frecuencias absolutas y relativas. Para las variables continuas se utilizaron medidas de dispersión y de tendencia central de acuerdo con su distribución. En casos de distribución normal se utilizaron promedios y desviaciones estándar, para variables con distribución no normal se utilizaron medianas y rangos. Se estimó la densidad de incidencia para enfermedad e infección por citomegalovirus, tomando como numerador el número de eventos y como denominador el tiempo a riesgo de cada paciente hasta la fecha en que culminó el seguimiento, tuvo el último control en la institución o hasta la muerte. El análisis se realizó en el programa STATA 12.0 .

\section{Criterios de inclusión}

Pacientes mayores de 18 años trasplantados renales de donante vivo y cadavérico en un centro de trasplante en el periodo 2004-2015.

\section{Criterios de exclusión}

- Paciente que no tenga datos de seguimiento en este centro de trasplante

- Pacientes con menos de seis meses de trasplante a la fecha final del estudio.

- Para efectos de este trabajo, se emplearon las siguientes definiciones:

- Trasplante renal riesgo intermedio para CMV: estado serológico usando IgG para CMV, todos los receptores con IgG positiva $(\mathrm{R}+)$.

- Riesgo Alto para CMV: D+/R-

- Riesgo Bajo para CMV: D-/R-

- Infección por CMV: positividad en la reacción en cadena de la polimerasa (PCR) para CMV en ausencia de síntomas (11).

- Enfermedad por CMV: Positividad en la PCR para CMV con presencia de síntomas, sea síndrome viral con fiebre, malestar, leucopenia o invasiva (11).

\section{Resultados}

Se realizaron 254 trasplantes renales desde enero $1^{\circ}$. de 2004 hasta 31 diciembre de 2015; de éstos 252 cumplieron los criterios de inclusión. La seroprevalencia para Ig G se distribuyó en: riesgo intermedio $92.4 \%$ (233 pacientes) y $7.5 \%$ riesgo alto (19 pacientes), no se encontraron pacientes de bajo riesgo en esta cohorte. Doscientos cincuenta pacientes recibieron profilaxis universal con valganciclovir durante 90 días y dos pacientes clasificados como riesgo alto se extendieron a 180 días. Se identificaron 19 casos, 13 con infección $(5.1 \%)$ y seis con enfermedad (2.3\%) (Tabla 1$)$.

La tasa de infección 10.08 casos por 1000 pacientes/año y la tasa de enfermedad 5.88 por 1000 pacientes/año. Se presentaron dos casos de infección temprana, antes de los
Tabla 1. Datos demográficos.

\begin{tabular}{|l|c|c|}
\hline & Infección (13) & Enfermedad (6) \\
\hline Masculino/femenino & $6 / 7$ & $5 / 1$ \\
\hline Edad diagnóstico (años) $(x)$ & $49.5(19-79$ a) & $47.5(19-74 \mathrm{a})$ \\
\hline Riesgo de CMV & & 1 \\
\hline Riesgo alto & 1 & 5 \\
\hline Riesgo intermedio & 12 & 2 \\
\hline Timoglobulina & 5 & 6 \\
\hline Profilaxis tres meses & 12 & 0 \\
\hline Profilaxis seis meses & 1 & 3 \\
\hline Gastrointestinal & - & 2 \\
\hline Pulmonar & - & 2 \\
\hline Retina & - & 3 \\
\hline Síndrome CMV & - & 3 \\
\hline Ganciclovir & 0 & $88( \pm 74.5)$ \\
\hline Valganciclovir & 13 & $650.3 \pm 481.7$ \\
\hline Tiempo tratamiento (días) (DE) & $44.8( \pm 73.8)$ & $1780( \pm 2552)$ \\
\hline Tiempo al diagnóstico (días) & $417 \pm 479$ & 2 \\
\hline Infección/enfermedad & & \\
\hline Diagnóstico & 13 & \\
\hline PCR por CMV & $04573( \pm 272848)$ & \\
\hline Antigenemia pp65 & 4 & \\
\hline Número de copias por PCR & & \\
\hline Rechazo celular & & \\
\hline
\end{tabular}

100 días y tres casos de enfermedad tardía, después de un año de trasplante.

El compromiso gastrointestinal fue el más frecuente (tres de seis pacientes), manifestándose como colitis, seguido por el compromiso pulmonar que se presentó como neumonitis por CMV (dos de seis pacientes).

El efecto adverso asociado al tratamiento que se presentó con mayor frecuencia fue leucopenia en $25 \%$ de los casos. La mortalidad fue $5 \%$, correspondiente a un paciente con compromiso gastrointestinal, dado por úlceras gastrointestinales.

\section{Discusión}

La infección por CMV es la infección viral que se presenta con mayor frecuencia en el postrasplante renal. La distribución de riesgo intermedio y alto para reactivación de infección en el postrasplante, es similar a la reportada en estudios a nivel nacional e internacional $(2,5)$. Ningún paciente fue de bajo riesgo, por ello todos los pacientes recibieron profilaxis universal. La densidad de incidencia de infección y enfermedad por CMV fue baja, lo que probablemente se explica por el uso de profilaxis universal. El medicamento utilizado para este fin, fue valganciclovir, de acuerdo con las guías internacionales. Dada la baja frecuencia de eventos, no fue posible establecer asociación con la terapia de inducción utilizada.

El tiempo de aparición de la infección fue en promedio 1.3 años postrasplante, dos casos se presentaron antes de terminar la profilaxis universal. Dado el diseño del estudio, no se lograron establecer factores asociados con la aparición de infección durante la fase de profilaxis. El diagnóstico en 
la mayoría de los casos se hizo con carga viral por PCR, que es el estándar de oro, sólo en un caso, el diagnóstico se hizo con antígeno pp65, ya que en ese momento no estaba disponible la PCR. En un caso de enfermedad, con compromiso gastrointestinal, confirmado por colonoscopia (úlcera en ciego) y patología (efectos citopáticos por CMV), la carga viral fue negativa. Recordamos que en casos de enfermedad que afecta tracto gastrointestinal o retina, la carga viral en sangre puede ser negativa y ello no descarta el diagnóstico. Esta es una infección que conlleva mayor morbimortalidad, por ello debe estar dentro de los diagnósticos diferenciales de patologías infecciosas en el paciente inmunosuprimido, la mortalidad en esta cohorte fue $5 \%$.

Este estudio tiene las limitaciones propias de un estudio retrospectivo, corresponde a un solo centro, lo que puede limitar la generalización de los resultados. Dada la baja frecuencia de eventos, no fue posible establecer asociaciones.

\section{Conclusiones}

En la cohorte de pacientes trasplantados, la densidad de incidencia de infección-enfermedad por CMV en pacientes trasplantados renales fue de 10.08 casos y 5.88 casos por 1000 pacientes/año, respectivamente. Estas tasas son menores a las reportados en la literatura, el uso de profilaxis universal en todos los pacientes puede contribuir a estos hallazgos.

\section{Referencias}

1. James Díaz-Betancur, Jorge Enrique Henao FAJ. Efectos de la infección y la enfermedad por citomegalovirus en receptores de trasplante renal. Acta Med Colomb. 2012;37:131-137.

2. Arias-Murillo YR, Osorio-Arango K, Cortés JA. Seroprevalencia de citomegalovirus en donantes de órganos y receptores de trasplante renal, Colombia, 2010-2014. Biomédica. 2016;36:24.

3. De Keyzer K, Van Laecke S, Peeters P, Vanholder R. Human cytomegalovirus and kidney transplantation: A clinician's update. Am J Kidney Dis. 2011;58(1):118126. doi:10.1053/j.ajkd.2011.04.010.

4. Gutiérrez E, Hernández E, Praga EMM.Afectación gastrointestinal severa por CMV tardío : la importancia del tratamiento precoz. Nefrología. 2007;27(6):779780 .

5. Ramanan P, Razonable RR. Cytomegalovirus infections in solid organ transplantation: a review. Infect Chemother. 2013;45(3):260-271. doi:10.3947/ ic.2013.45.3.260.

6. Giakoustidis D, Antoniadis A, Fouzas I, et al. Prevalence and clinical impact of cytomegalovirus infection and disease in renal transplantation: Ten years of experience in a single center. Transplant Proc. 2012;44(9):2715-2717.

7. Razonable RR. Management strategies for cytomegalovirus infection and disease in solid organ transplant recipients. Infect Dis Clin North Am. 2013;27(2):317-342. doi:10.1016/j.idc.2013.02.005.

8. Witzke O, Hauser I a., Bartels M, Wolf G, Wolters H, Nitschke M. Valganciclovir Prophylaxis Versus Preemptive Therapy in Cytomegalovirus-Positive Renal Allograft Recipients: 1-Year Results of a Randomized Clinical Trial. Transplantation. 2012;93(1):61-68. doi:10.1097/TP.0b013e318238dab3.

9. Luan FL, Kommareddi M, Ojo AO. Universal prophylaxis is cost effective in cytomegalovirus serology-positive kidney transplant patients. Transplantation. 2011;91(2):237-244.

10. Kotton CN, Kumar D, Caliendo AM, et al. Updated international consensus guidelines on the management of cytomegalovirus in solid-organ transplantation. Transplantation. 2013;96(4):333-360. doi:10.1097/TP.0b013e31829df29d.

11. Cortés JA, Yomayusa N, Arias YR, et al. Consenso colombiano para la estratificación, diagnóstico, tratamiento y prevención de la infección por citomegalovirus en pacientes adultos con trasplante renal. Infectio. 2015. doi:10.1016/j. infect.2015.10.005 